\title{
Analysis of Ball-Type Constant-Velocity Joints Based on Dynamics*
}

\author{
Kei KIMATA** ${ }^{* *}$ Haruo NAGATANI*** and Masayuki IMOTO***
}

\begin{abstract}
Universal joints, which transmit torque through the balls guided in such a manner that they always lie in the plane bisecting the angle between the driving-shaft and driven-shaft, are called ball-type constant-velocity joints. The authors presented papers on the analysis of this type of universal joint based on statics neglecting the friction force between their components. To obtain more precise estimations of the internal forces and the moments, it appears to be important to evaluate the effect of the friction between the parts. Furthermore, grasping the relative motions of the parts, such as rolling of the balls on the tracks, is beyond the scope of static analysis. In this study, the authors analyze the motions of each part based on dynamics, taking account of the friction between the parts. The analysis results in simultaneous differential equations, which can be solved numerically using an electronic computer.
\end{abstract}

Key Words: Machine Element, Universal Joints, Constant Velocity Joints, Ball-Type, Angular Contact, Contact Force, Friction, Dynamics, Analysis

\section{Introduction}

Universal joints which transmit torque and rotational motion of the shaft through the balls whose center is constrained on the plane bisecting the crossed axes angle between the driving and driven shafts (hereafter referred to as homo-kinetic plane) are called ball-type constantvelocity joints. In the previous papers ${ }^{(1),(2)}$, the authors proposed a method for analyzing the forces acting on the inside of the constant velocity universal joint of this type based on statics with neglecting the frictional forces, and presented the examples of the results of numerical computations.

On the other hand, one of the authors investigated the effects of the frictional forces acting on the inside of constant velocity joints and pointed out that frictional force has significant effects on the distribution of the forces acting on the inside as well as on the power transmission efficiency of the ball-type constant-velocity joints ${ }^{(3)}$.

* Received 4th November, 2003 (No. 02-0216). Japanese Original: Trans. Jpn. Soc. Mech. Eng., Vol.69, No.678, C (2003), pp.478-486 (Received 25th February, 2002)

** Former Employee of NTN Corporation, 1618 Hachisuka, Miwa-cho, Ama-gun, Aichi 490-1225, Japan

*** NTN Corporation, Automotive Engineering Dept. Automotive Sales Headquarters, 1578 Higashi-kaizuka, Iwatashi, Shizuoka 438-8510, Japan.

E-mail: haruo_nagatani@ntn.co.jp
In order to grasp more accurately the characteristics of ball-type constant-velocity joints, the forces acting on the inside, and the relative motion of the parts, it is considered necessary to carry out the analysis based on dynamics taking the frictional forces into considerations. In this paper, the motion of each part of the ball-type constantvelocity joint is expressed by equations of motion in which frictional forces are taken into account, and simultaneous ordinary differential equations are derived.

\section{Universal Joint of Object Under Analysis and Pre- requisites for Analysis}

The ball-type constant-velocity joint analyzed in this paper has the following features:

(1) It has an inner race coupled with one shaft and an outer race coupled with the other shaft, and the balls are constrained by the tracks provided in the inner and outer races and the cage.

(2) The track center line of the inner race and that of the outer race are symmetrical about the homo-kinetic plane, and the contact between the track and the ball is of angular type.

( 3 ) Each center plane of the cage window is flushed with the homo-kinetic plane, and the planes in contact with the ball are flat.

Ball-type constant-velocity joints equipped with the above-mentioned features are, for example, Ball fixed joint (BJ), Undercut free joint (UJ), Double offset joint 


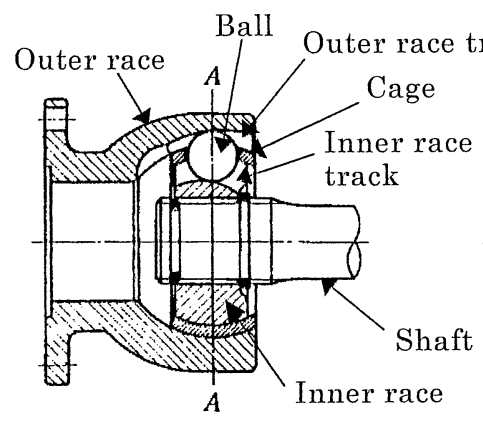

Section B-B

(a) Longitudinal section

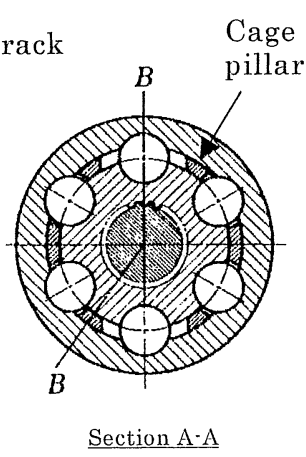

(b) Cross section
Fig. 1 Ball fixed joint (BJ)

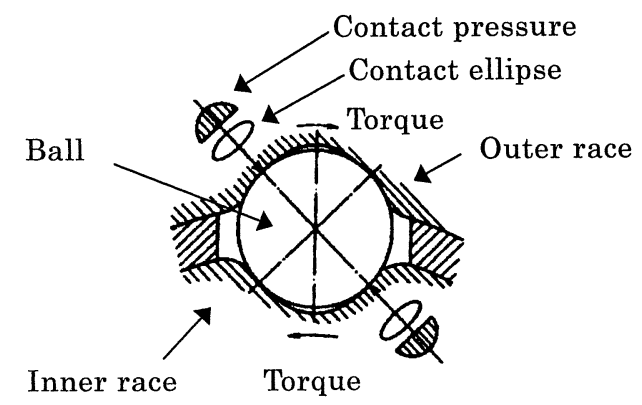

Fig. 2 Cross-sectional shape of tracks

(DOJ), and Cross groove joint $(\mathrm{LJ})^{(4)}$. Figure 1 shows BJ and Fig. 2 shows the cross section of the track of a balltype constant-velocity joint.

For simplicity, analysis is performed based on the following prerequisites:

(1) As for the deformation of the parts, only the local elastic deformation at the mutual contact area of the ball, inner race, outer race, and cage is taken into consideration.

( 2 ) Frictional force proportional to the contact surface pressure is applied at the contact area between parts.

(3) The axis of the outer race is supported by fixed bearings, and the end of the inner race axis is connected to another universal joint.

(4) The rotational speed of the outer race is constant.

\section{Coordinate System and Symbols}

In the same manner as used in Ref.(1), a right-handed orthogonal coordinate system is set. In the coordinates, the $x-y$ plane includes the inner race and outer race axis of the universal joint, the origin coincides with the intersection point of both axes, and the homo-kinetic plane coincides with the $y-z$ plane. Figure 3 shows this coordinate system. The cage axis either coincides with the $x$-axis or is parallel to it. In case that the cage has offset $(h)$, it is considered to be in the positive state if the center of the inner sphere of the cage $\left(\mathrm{O}_{1}\right)$ is at the $x>0$ side and the center of the outer sphere of the cage $\left(\mathrm{O}_{2}\right)$ is at the $x<0$ side. It is considered that the center of gravity $\left(G_{c}\right)$ of the cage should be on $\overline{\mathrm{O}_{1} \mathrm{O}_{2}}$. Operating angle $(2 \theta)$ is consid-

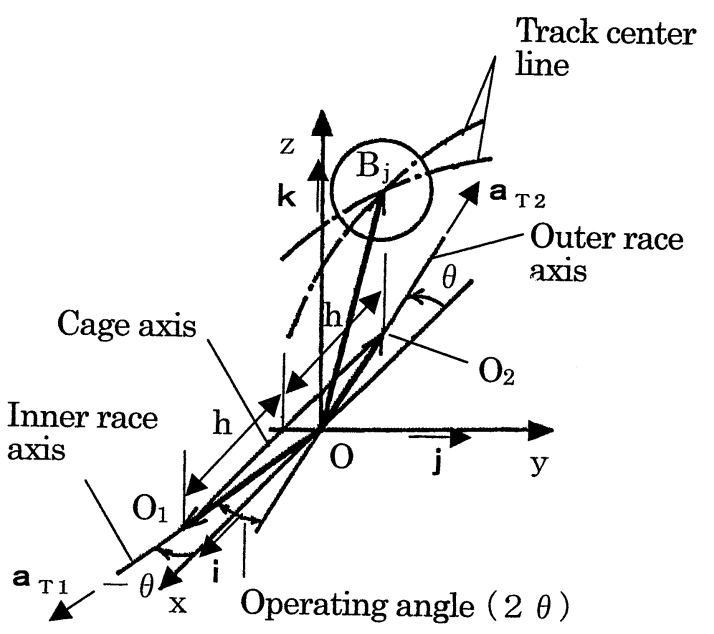

Fig. 3 Coordinates

ered to be in the positive state when the outer race axis is inclined in the right-hand-screw rotation direction that advances in the $z$-axis direction, and the inner race axis is inclined in the direction opposite to it.

Key symbols are summarized below.

$\boldsymbol{a}:$ Unit vector

$\boldsymbol{B}$ : Position vector that denotes center of ball or center of curvature of track

$\boldsymbol{E}$ : Parallel displacement

$\boldsymbol{i}, \boldsymbol{j}, \boldsymbol{k}:$ Fundamental vector

$\boldsymbol{G}$ : Position vector that denotes center of gravity

$\boldsymbol{V}$ : Velocity vector on contact surface

$\boldsymbol{\eta}$ : Angular displacement vector of inner race axis normal to it

$\varepsilon$ : Angular displacement vector of cage

$\omega$ : Rotational speed

$\boldsymbol{\Omega}$ : Relative rotational displacement vector (in axial direction) of inner race against outer race

$d:$ Diameter of ball

$g$ : Clearance between inner/outer race and cage

$M:$ Mass

$n:$ Number of balls

$T:$ Torque

$\delta:$ Amount of elastic approach

$\Delta$ : Clearance between ball and inner/outer race track

$\phi$ : Rotation angle

$t$ : Time

Suffixes:

$a$ : Shaft

$B$ : Ball

$c:$ Cage

$G$ : Center of gravity

$j:$ Ball number

$k: k=1,2$ Inner race track

$k=3,4$ Outer race track

$k=5,6$ Ball guide-plane of cage

$k=7$ Pillar of cage 
Table 1 Forces and moments acting inside ball-type CVJ

\begin{tabular}{|c|c|c|c|c|c|}
\hline & \multicolumn{2}{|c|}{ Contact force } & \multirow[b]{2}{*}{$\begin{array}{c}\text { Frictional } \\
\text { force }\end{array}$} & \multicolumn{2}{|c|}{ Moment of frictional force } \\
\hline & Force & $\begin{array}{l}\text { Point of } \\
\text { Action }\end{array}$ & & Moment & Point of Action \\
\hline $\begin{array}{l}\text { Forces and moments from } \\
\text { inner race to ball }\end{array}$ & $\begin{array}{l}P_{1 j} \\
P_{2 j}\end{array}$ & $B_{j}+E_{B j}$ & $\begin{array}{l}Q_{1 j} \\
Q_{2 j}\end{array}$ & $\begin{array}{l}\mathbf{m}_{\mathrm{d} 1 \mathrm{j}} \\
\mathbf{m}_{\mathrm{d} 2 \mathrm{j}}\end{array}$ & $\begin{array}{l}B_{j}+E_{B j}+r_{1 j} \\
B_{j}+E_{B j}+r_{2 j}\end{array}$ \\
\hline $\begin{array}{l}\text { Forces and moments from } \\
\text { outer race to ball }\end{array}$ & $\begin{array}{l}-P_{3 j} \\
-P_{4 j} \\
\end{array}$ & $B_{j}+E_{B j}$ & $\begin{array}{l}Q_{3 j} \\
Q_{4 j}\end{array}$ & $\begin{array}{l}m_{\mathrm{d} 3 j} \\
m_{\mathrm{d} 4 j}\end{array}$ & $\begin{array}{l}B_{j}+E_{B j}+r_{3 j} \\
B_{j}+E_{B j}+r_{4 j}\end{array}$ \\
\hline $\begin{array}{l}\text { Forces and moments from } \\
\text { cage to ball }\end{array}$ & $\begin{array}{l}-P_{5 j} \\
-P_{6 j} \\
-P_{7 j}\end{array}$ & $B_{j}+E_{B j}$ & $\begin{array}{l}Q_{5 j} \\
Q_{6 j} \\
Q_{7 j}\end{array}$ & $\begin{array}{l}\mathbf{m}_{\mathrm{d} 5 \mathrm{j}} \\
\mathbf{m}_{\mathrm{d} 6 \mathrm{j}} \\
\mathbf{m}_{\mathrm{d} 7 \mathrm{j}}\end{array}$ & $\begin{array}{l}B_{j}+E_{B j}+r_{5 j} \\
B_{j}+E_{B j}+r_{6 j} \\
B_{j}+E_{B j}+r_{7 j}\end{array}$ \\
\hline $\begin{array}{l}\text { Forces and moments from } \\
\text { inner race to cage }\end{array}$ & $\mathbf{F}_{1}$ & $O_{1}+E_{1}$ & $\mathrm{H}_{1}$ & $\mathrm{~m}_{\mathrm{H} 1}$ & $O_{1}+E_{1}$ \\
\hline $\begin{array}{l}\text { Forces and moments from } \\
\text { cage to outer race }\end{array}$ & $\mathrm{F}_{2}$ & $\begin{array}{c}\mathrm{O}_{2}+\mathrm{E}_{\mathrm{Gc}}+ \\
\xi \times\left(\mathrm{O}_{2}-\mathrm{G}_{\mathrm{c}}\right)\end{array}$ & $\mathrm{H}_{2}$ & $\mathbf{m}_{\mathrm{H} 2}$ & $\begin{array}{c}\mathrm{O}_{2}+\mathrm{E}_{\mathrm{Gc}}+ \\
\xi \times\left(\mathrm{O}_{2}-\mathrm{G}_{\mathrm{c}}\right)\end{array}$ \\
\hline
\end{tabular}

$m: m=1$ Inner race $m=2$ Outer race

$T:$ Longitudinal direction of drive shaft

$x, y, z x, y, z:$ component

'(Prime) : Prime denotes the value after displacement Rotational tensor:

$\boldsymbol{A}_{x}(\phi)$ : Vector is turned about $x$-axis by angle $\phi$ $\boldsymbol{A}_{y}(\gamma)$ : Vector is turned about $y$-axis by angle $\gamma$ $\boldsymbol{A}_{z}(\theta)$ : Vector is turned about $z$-axis by angle $\theta$

Symbols relating to forces and moments acting on the inside of the universal joint are summarized in Table 1. Symbols other than those referred to above will be explained in the text.

\section{Relationship between Forces Acting on Each Part and Displacement}

\section{1 Forces acting on interface between ball and track}

4.1.1 Normal force Figure 4 shows a section that passes through the center of the ball $\left(B_{j}\right.$ or $\left.B_{j}^{\prime}\right)$ and is perpendicular to the tangential line of the track centerline $\left(\boldsymbol{a}_{t m j}\right.$ or $\left.\boldsymbol{a}_{t m j}^{\prime}\right)$ at this point. Line $\boldsymbol{a}_{k j}(k=1-4)$ is the common normal line at the contact point between the ball and track in the design. $\boldsymbol{B}_{j}, \boldsymbol{a}_{t m j}$, and $\boldsymbol{a}_{k j}$ are described in Ref.(1). While the ball type constant velocity joint is transmitting power, the inner race parallel displacement $\boldsymbol{E}_{1}$ and relative rotational displacement $\boldsymbol{\Omega}$, with regard to the outer race, are possible. The above vectors also change with regard to the reference vectors. The vectors after displacement can be expressed as follows:

$$
\begin{aligned}
& \boldsymbol{B}_{j}^{\prime}=\boldsymbol{B}_{j}+\boldsymbol{E}_{B j} \\
& \begin{cases}\boldsymbol{a}_{t m j}^{\prime}=\frac{\boldsymbol{a}_{t m j}+\boldsymbol{\Omega} \times \boldsymbol{a}_{t m j}}{\left|\boldsymbol{a}_{t m j}+\boldsymbol{\Omega} \times \boldsymbol{a}_{t m j}\right|} & (m=1) \\
\boldsymbol{a}_{t m j}^{\prime}=\boldsymbol{a}_{t m j} & (m=2)\end{cases}
\end{aligned}
$$

$\boldsymbol{B}_{k j}$ is the position vector which represents the center of curvature of the track in the section through the ball center and perpendicular to the track center line and can be expressed as

$$
\boldsymbol{B}_{k j}=\boldsymbol{B}_{j}+(-1)^{m+1} k_{m} \frac{d}{2} \boldsymbol{a}_{k j}
$$

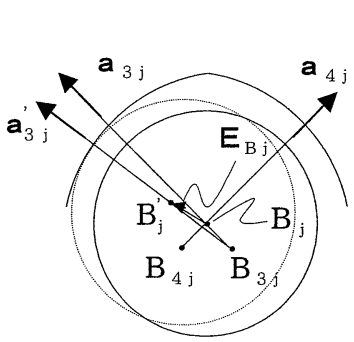

(a) Outer race track

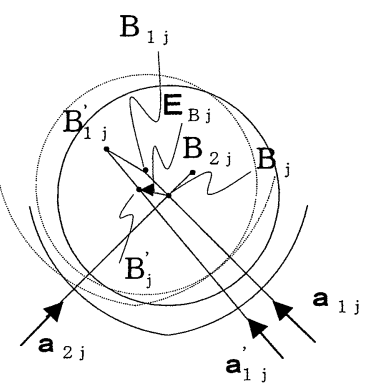

(b) Inner race track
Fig. 4 Section through ball center and perpendicular to track center line

—Standard position,- - - After displacement

in which $k_{m}$ is curvature ratio (curvature radius of track section/ball radius-1). The center of curvature after displacement can be expressed as

$$
\begin{cases}\boldsymbol{B}_{k j}^{\prime}=\boldsymbol{B}_{k j}+\boldsymbol{E}_{1}+\boldsymbol{\Omega} \times \boldsymbol{B}_{k j} & (k=1,2) \\ \boldsymbol{B}_{k j}^{\prime}=\boldsymbol{B}_{k j} & (k=3,4)\end{cases}
$$

$\boldsymbol{a}_{k j}^{\prime}$ is a unit vector on the line that is drawn from point $B_{j}^{\prime}$ and crosses perpendicularly the straight line in the $\boldsymbol{a}_{t m j}^{\prime}$ direction passing through point $B_{k j}^{\prime}$. This is expressed as

$$
\begin{gathered}
\boldsymbol{a}_{k j}^{\prime}=(-1)^{m+1} \frac{\boldsymbol{B}_{k j}^{\prime}-\boldsymbol{B}_{j}^{\prime}-\left\{\left(\boldsymbol{B}_{k j}^{\prime}-\boldsymbol{B}_{j}^{\prime}\right) \cdot \boldsymbol{a}_{t m j}^{\prime}\right\} \boldsymbol{a}_{t m j}^{\prime}}{\left|\boldsymbol{B}_{k j}^{\prime}-\boldsymbol{B}_{j}^{\prime}-\left\{\left(\boldsymbol{B}_{k j}^{\prime}-\boldsymbol{B}_{j}^{\prime}\right) \cdot \boldsymbol{a}_{t m j}^{\prime}\right\} \boldsymbol{a}_{t m j}^{\prime}\right|} \\
(k=1,2,3,4)
\end{gathered}
$$

The amount of interference between the ball and the inner and outer race tracks can be expressed as

$$
\begin{cases}\delta_{k j}=\left\{\boldsymbol{E}_{1}+\boldsymbol{\Omega} \times \boldsymbol{B}_{j}-\boldsymbol{E}_{B j}\right\} \cdot \boldsymbol{a}_{k j}^{\prime}-\Delta_{k j} & (k=1,2) \\ \delta_{k j}=\boldsymbol{E}_{B j} \cdot \boldsymbol{a}_{k j}^{\prime}-\Delta_{k j} & (k=3,4)\end{cases}
$$

If this amount of interference is absorbed by local elastic deformation, a contact force is generated. This force is expressed by ${ }^{(5)}$

$$
\left\{\begin{array}{ll}
\boldsymbol{P}_{k j}=K_{k j} \delta_{k j}^{3 / 2} \boldsymbol{a}_{k j}^{\prime} & \delta_{k j}>0 \\
\boldsymbol{P}_{k j}=0 & \delta_{k j} \leq 0
\end{array} \quad(k=1,2,3,4)\right.
$$

in which $K_{k j}$ is a factor depending on the elastic constant of the material and the curvatures of the contact surfaces. 


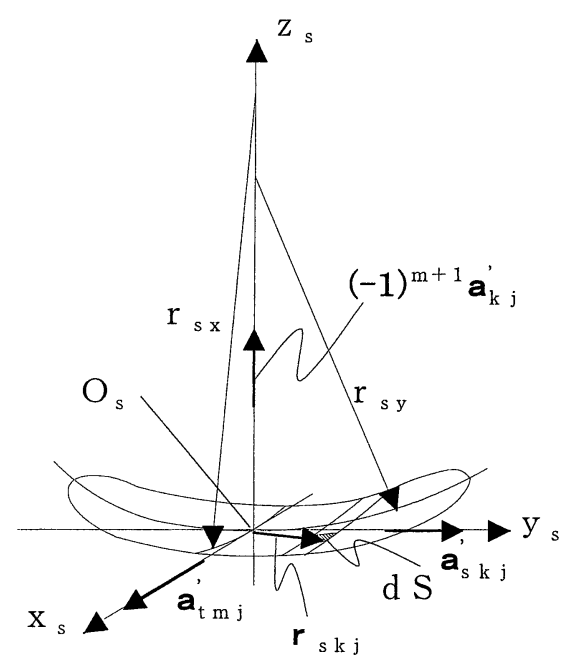

Fig. 5 Contact ellipse and local coordinates

\subsubsection{Frictional forces and moments by frictional}

forces Figure 5 shows a contact ellipse generated when normal force $P_{k j}$ is acting at the interface between the track and ball. The position vector from the center of the ball $B_{j}^{\prime}$ until the center of the contact ellipse $\mathrm{O}_{\mathrm{s}}$ can be expressed as

$$
\boldsymbol{r}_{k j}=(-1)^{m} \frac{d}{2} \boldsymbol{a}_{k j}^{\prime}\left(\begin{array}{ll}
m=1 & k=1,2 \\
m=2 & k=3,4
\end{array}\right)
$$

The origin of the local coordinates shown in Fig. 5 coincides with the center of the contact ellipse $\mathrm{O}_{\mathrm{s}}$, and the $x_{s}$, $y_{s}$, and $z_{s}$ axes coincides with $\boldsymbol{a}_{t m j}^{\prime}, \boldsymbol{a}_{s k j}^{\prime}$, and $(-1)^{m+1} \boldsymbol{a}_{k j}^{\prime}$, respectively. $\boldsymbol{a}_{s k j}^{\prime}$ is a unit tangential vector to the track surface and perpendicular to $\boldsymbol{a}_{t m j}^{\prime} . \boldsymbol{a}_{s k j}^{\prime}$ is given by

$$
\boldsymbol{a}_{s k j}^{\prime}=(-1)^{m+1} \boldsymbol{a}_{k j}^{\prime} \times \boldsymbol{a}_{t m j}^{\prime}
$$

The method of calculating the dimensions of a contact ellipse and the surface pressure distribution in the contact ellipse is shown in Ref.(5). The ball is designated as substance I, and the inner and outer race tracks are designated as substance II. The curvatures of these substances in the $x_{s}-z_{s}$ plane are designated as $\rho_{\mathrm{I} x}$ and $\rho_{\mathrm{II} x}$, and the curvatures in the $y_{s}-z_{s}$ plane are designated as $\rho_{\mathrm{I} y}$ and $\rho_{\mathrm{II} y}$. Then the curvature of the contact ellipse is given by ${ }^{(6)}$

$$
\frac{1}{\gamma_{s x, y}}=\frac{1}{2}\left(\rho_{\mathrm{I} x, y}-\rho_{\mathrm{II} x, y}\right)
$$

Position vector $\boldsymbol{r}_{s k j}$ from origin $\mathrm{O}_{\mathrm{s}}$ of the local coordinates until a point on the contact ellipse $\left(x_{s}, y_{s}, z_{s}\right)$ is given by

$$
\boldsymbol{r}_{s k j} \fallingdotseq x_{s} \boldsymbol{a}_{t m j}^{\prime}+y_{s} \boldsymbol{a}_{s k j}^{\prime}+(-1)^{m+1} \frac{1}{2}\left(\frac{x_{s}^{2}}{r_{s x}}+\frac{y_{x}^{2}}{r_{s y}}\right) \boldsymbol{a}_{k j}^{\prime}
$$

The angular velocities of the inner and outer race are expressed as

$$
\begin{aligned}
& \omega_{1}=(\omega+\dot{\Omega}) \boldsymbol{a}_{T 1} \\
& \omega_{2}=-\omega \boldsymbol{a}_{T 2}
\end{aligned}
$$

Velocity $\boldsymbol{V}_{k j}$ of the track surface at the point $\left(x_{s}, y_{s}\right.$, $\left.z_{s}\right)$ in the contact ellipse is given by

$$
\begin{aligned}
& \boldsymbol{V}_{k j}=\omega_{1} \times\left(\boldsymbol{B}_{j}+\boldsymbol{E}_{B j}+\boldsymbol{r}_{k j}+\boldsymbol{r}_{s k j}-\boldsymbol{E}_{1}\right)+\dot{\boldsymbol{E}}_{1} \quad(k=1,2) \\
& \boldsymbol{V}_{k j}=\omega_{2} \times\left(\boldsymbol{B}_{j}+\boldsymbol{E}_{B j}+\boldsymbol{r}_{k j}+\boldsymbol{r}_{s k j}\right) \quad(k=3,4)
\end{aligned}
$$

Similarly, the velocity on the ball surface is given by

$$
\boldsymbol{V}_{B k j}=\omega_{B j} \times\left(\boldsymbol{r}_{k j}+\boldsymbol{r}_{s k j}\right)+\dot{\boldsymbol{B}}_{j}+\dot{\boldsymbol{E}}_{B j} \quad(k=1,2,3,4)
$$

Unit vector $\boldsymbol{a}_{V}$, which represents the direction of the track surface relative velocity with regard to the ball surface at the point of $\left(x_{s}, y_{s}, z_{s}\right)$, is given by

$$
\boldsymbol{a}_{V}=\frac{\boldsymbol{V}_{k j}-\boldsymbol{V}_{B k j}}{\left|\boldsymbol{V}_{k j}-\boldsymbol{V}_{B k j}\right|} \quad(k=1,2,3,4)
$$

Therefore, the frictional force acting from the track on the ball in the contact ellipse generated by the contact of the ball and the track is

$$
\boldsymbol{Q}_{k j}=\iint \sigma p\left(x_{s}, y_{s}\right) \boldsymbol{a}_{V} d x_{s} d y_{s} \quad(k=1,2,3,4)
$$

in which $\mathrm{p}\left(x_{s}, y_{s}\right)$ denotes contact surface pressure, and $\sigma$ denotes coefficient of friction.

The moment of the frictional force acting on the ball relating to the origin of the absolute coordinates can be expressed as

$$
\begin{aligned}
\boldsymbol{m}_{B k j}= & \iint_{\times\left\{\sigma p\left(x_{s}, y_{s}\right) \boldsymbol{a}_{V}\right\} d x_{s} d y_{s}}\left(\boldsymbol{E}_{B j}+\boldsymbol{r}_{k j}+\boldsymbol{r}_{s k j}\right)
\end{aligned}
$$

Equation (19) can be rewritten using Eq. (18) as

$$
\begin{aligned}
& \boldsymbol{m}_{B k j}=\left(\boldsymbol{B}_{j}+\boldsymbol{E}_{B j}+\boldsymbol{r}_{k j}\right) \times \boldsymbol{Q}_{k j}+\boldsymbol{m}_{d k j} \quad(k=1,2,3,4) \\
& \boldsymbol{m}_{d k j}=\iint \sigma p\left(x_{s}, y_{s}\right)\left(\boldsymbol{r}_{s k j} \times \boldsymbol{a}_{V}\right) d x_{s} d y_{x} \quad(k=1,2,3,4)
\end{aligned}
$$

in which $\boldsymbol{m}_{d k j}$ denotes the moment around the origin of the local coordinates.

\subsection{Force acting on the interface between ball and cage}

4.2.1 Force acting on ball guide-plane As for the displacement of the cage, the parallel displacement of the center of gravity $\boldsymbol{E}_{G} c$ and the angular displacement around the center of gravity $\xi$ are possible. $\xi$ can be expressed as

$$
\boldsymbol{\xi}=\xi_{y} \boldsymbol{j}+\xi_{z} \boldsymbol{k}
$$

Vector $\boldsymbol{a}_{k j}=(-1)^{k+1} \boldsymbol{i}(k=5,6)$, the normal to the ball guide-plane of the cage window, is changed due to $\xi$ as

$$
\boldsymbol{a}_{k j}^{\prime}=\frac{(-1)^{k+1}(\boldsymbol{i}+\boldsymbol{\xi} \times \boldsymbol{i})}{|\boldsymbol{i}+\boldsymbol{\xi} \times \boldsymbol{i}|} \quad(k=5,6)
$$

The position vector from the center of the ball until the contact point with the ball guide-plane of the cage window is

$$
\boldsymbol{r}_{k j}=\frac{d}{2} \boldsymbol{a}_{k j}^{\prime} \quad(k=5,6)
$$


The amount of interference at this contact point is

$$
\begin{gathered}
\delta_{k j}=\boldsymbol{a}_{k j}^{\prime} \cdot\left[\boldsymbol{E}_{B j}-\boldsymbol{E}_{G C}-\boldsymbol{\xi} \times\left(-\boldsymbol{G}_{C}+\boldsymbol{B}_{j}\right)\right]-\Delta_{k j} \\
(k=5,6)
\end{gathered}
$$

Contact force $\boldsymbol{P}_{k j}(k=5,6)$ generated by this interference is obtained by substituting suffixes $k=5,6$ into Eq. (7).

The curvature radius of the contact ellipse (a circle in this case) generated by this contact force is obtained by Eq. (10) to be $d$. Local coordinates $\left(x_{s}, y_{s}, z_{s}\right)$ whose origin is at the center of this contact circle are set up. The direction of the $z_{s}$ axis is adjusted to $-\boldsymbol{a}_{k j}^{\prime}(k=5,6)$. Suppose that the unit vectors along the $x_{s}$ axis and $y_{s}$ axis are expressed by $\boldsymbol{a}_{t k j}^{\prime}$ and $\boldsymbol{a}_{s k j}^{\prime}(k=5,6)$, respectively. Then the position vector $\boldsymbol{r}_{s k j}(k=5,6)$ from the origin of the local coordinates until a point on the contact surface $\left(x_{s}, y_{s}, z_{s}\right)$ is given by Eq. (11) as

$$
\boldsymbol{r}_{s k j}=x_{s} \boldsymbol{a}_{t k j}^{\prime}+y_{s} \boldsymbol{a}_{s k j}^{\prime}-\frac{1}{2 d}\left(x_{s}^{2}+y_{s}^{2}\right) \boldsymbol{a}_{k j}^{\prime} \quad(k=5,6)
$$

The angular velocity of the cage is expressed as

$$
\omega_{c}=\dot{\phi}_{c} a_{5 j}^{\prime}+\dot{\xi}
$$

Velocity $\boldsymbol{V}_{k j}(k=5,6)$ of the ball guide-plane at the point $\left(x_{s}, y_{s}, z_{s}\right)$ on the contact surface is given by

$$
\begin{gathered}
\boldsymbol{V}_{k j}=\boldsymbol{\omega}_{c} \times\left(\boldsymbol{B}_{j}+\boldsymbol{E}_{B j}+\boldsymbol{r}_{k j}+\boldsymbol{r}_{s k j}-\boldsymbol{G}_{c}-\boldsymbol{E}_{G c}\right)+\dot{\boldsymbol{E}}_{G C} \\
(k=5,6)
\end{gathered}
$$

$\boldsymbol{V}_{B k j}, \boldsymbol{a}_{V}, \boldsymbol{Q}_{k j}, \boldsymbol{m}_{B k j}$, and $\boldsymbol{m}_{d k j}(k=5,6)$ are obtained by substituting suffixes $k=5,6$ into Eqs. (16) $-(18),(20)$, (21), respectively.

4.2.2 Force acting on cage pillar Figure 6 shows a section obtained by cutting the cage at the plane perpendicular to the cage axis including the center of the window. The symbols used in this illustration are as

$b_{c j}: 1 / 2$ of length of window

$C_{j}$ : Center of the window

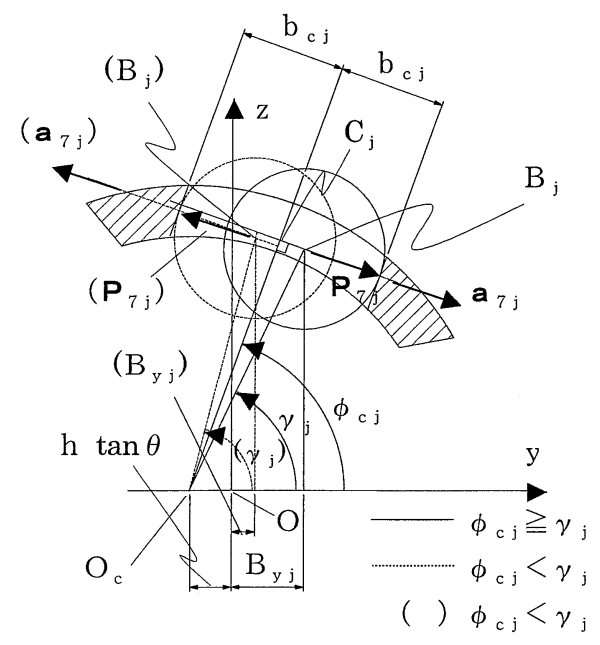

Fig. 6 Contact between ball and pillar of cage $\boldsymbol{a}_{7 j}$ : Normal vector to cage pillar (unit vector)

$\phi_{c j}$ : Rotation angle of cage window with regard to $y$ axis

$\mathrm{O}_{c}$ : Intersection point of cage axis and center plane of window (homo-kinetic plane)

$\gamma_{j}$ : Angle between $y$-axis and $\overline{\mathrm{O}_{c} \mathrm{~B}_{j}}$

$\boldsymbol{a}_{7 j}$ : can be expressed as

$$
\boldsymbol{a}_{7 j}=\operatorname{sign}\left(\gamma_{j}-\phi_{c j}\right)\left(-\sin \phi_{c j} \boldsymbol{j}+\cos \phi_{c j} \boldsymbol{k}\right)
$$

After displacement,

$$
\boldsymbol{a}_{7 j}^{\prime}=\frac{\boldsymbol{a}_{7 j}+\boldsymbol{\xi} \times \boldsymbol{a}_{7 j}}{\left|\boldsymbol{a}_{7 j}+\boldsymbol{\xi} \times \boldsymbol{a}_{7 j}\right|}
$$

Displacement $\boldsymbol{E}_{c}$ of $\mathrm{O}_{c}$ can be expressed as

$$
\boldsymbol{E}_{c}=\boldsymbol{E}_{G c}+\boldsymbol{\xi} \times\left(-h \tan \theta \boldsymbol{j}-G_{c}\right)
$$

$\gamma_{j}$ can be obtained from the following relationships.

$$
\begin{aligned}
& A_{y j}=B_{y j}+h \tan \theta+E_{B y j}-E_{c y} \\
& A_{z j}=B_{z j}+E_{B z j}-E_{c z} \\
& \tan \gamma_{j}=A_{z j} / A_{y j}
\end{aligned}
$$

The amount of interference $\delta_{7 j}$ between the ball and the cage pillar is given by

$$
\delta_{7 j}=\sqrt{A_{y j}^{2}+A_{z j}^{2}} \sin \left|\phi_{c j}-\gamma_{j}\right|+\frac{d}{2}-b_{c j}
$$

Contact force $\boldsymbol{P}_{7 j}$ loaded between the ball and the cage pillar can be expressed by substituting $k=7$ into Eq. (7).

The position vector from the center of the ball until the contact point between the ball and cage pillar is given by $\boldsymbol{r}_{7 j}=(d / 2) \boldsymbol{a}_{7 j}^{\prime}$.

Local coordinates $\left(x_{s}, y_{s}, z_{s}\right)$ for which the origin is at the center of the contact circle and the $z_{s}$ axis agrees with $-\boldsymbol{a}_{7 j}$ are set up. The unit vectors on the $x_{s}$ axis and $y_{s}$ axis are designated as $\boldsymbol{a}_{t 7 j}^{\prime}$ and $\boldsymbol{a}_{s 7 j}^{\prime}$, respectively. $\boldsymbol{r}_{s 7 j}, \boldsymbol{V}_{7 j}$, $\boldsymbol{V}_{B 7 j}, \boldsymbol{a}_{V}, \boldsymbol{Q}_{7 j}, \boldsymbol{m}_{B 7 j}, m_{d 7 j}$ are expressed by Eqs. (26), (28), (16) - (18), (20), (21) respectively, by substituting $k=7$.

\subsection{Force acting at the interface between cage and inner/outer races}

The cage and the inner race and outer race of the balltype constant-velocity joint in practical use fit between spherical surfaces or between a spherical surface and a cylindrical surface. Since tracks are provided on the inner race outer sphere and the outer race inner sphere, actual contact takes place in the narrow width portion between tracks even in the case of a fit between spherical surfaces.

Palmgren introduced the following approximate expression $^{(7)}$ for the amount of elastic approach $(\mathrm{mm})$ for the case where steel rollers with $l[\mathrm{~mm}]$ length are made to contact in parallel to produce load $P \mathrm{~N}$.

$$
\delta=3.84 \times 10^{-5} \frac{P^{0.9}}{l^{0.8}}
$$

Since the roller diameter is not included in this equation, the effects of the curvature radius of the contact surface on the amount of elastic approach for line contact is 


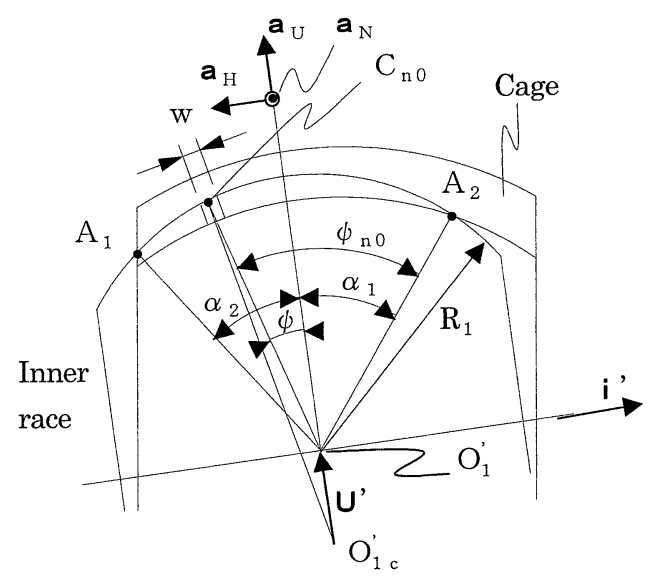

Fig. 7 Contact between inner race and cage

less significant. In Eq. (36), Harris replaced the roller with an assembly consisting of as many as $n_{1}$ thin disks with $w \mathrm{~mm}$ thickness and introduced the following equation assuming that one thin disk supports a load of $q \mathrm{~N}^{(8)}$.

$$
q=\left(\frac{\delta w^{0.8}}{3.84 \times 10^{-5} n_{1}^{0.1}}\right)^{\frac{1}{0.9}}
$$

Harris used this equation for the analysis of roller bearings with misalignment. This equation is also used in this paper. Namely, it is assumed that the contact area with narrow spherical fit width can be handled approximately as a line contact to use the method proposed by Harris.

Figure 7 shows a section obtained by cutting the inner race and cage to fit spherically at the plane that includes the inner race axis and forms an angle of $\phi_{j}+\pi / n$ with the $x y$-plane. In Fig. $7, \boldsymbol{i}^{\prime}$ denotes the inner race axis, $\mathrm{O}^{\prime}{ }_{1}$ denotes the spherical center of the inner race, and $\mathrm{O}^{\prime}{ }_{1 c}$ denotes the foot of the perpendicular drawn from the center of the inner sphere of the cage to this plane. Interference takes place at part $\widehat{\mathrm{A}_{1} \mathrm{~A}_{2}}$, and the contact force generated while this interference is absorbed by elastic deformation is obtained hereunder. Due to the limitations of space, the outline of the analysis is shown.

Suppose $\boldsymbol{a}_{N}$ denotes a unit vector perpendicular to the paper, then,

$$
\boldsymbol{a}_{N}=\boldsymbol{A}_{z(-\theta)} \boldsymbol{A}_{x}\left(\phi_{j}+\frac{\pi}{n}\right)(-\boldsymbol{k})
$$

Vector $\boldsymbol{U}$ from the center of the cage inner sphere $\mathrm{O}_{1 c}$ until $\mathrm{O}_{1}^{\prime}$ is given by

$$
\boldsymbol{U}=\boldsymbol{E}_{1}-\boldsymbol{E}_{G C}-\boldsymbol{\xi} \times\left(\boldsymbol{O}_{1}-\boldsymbol{G}_{c}\right)
$$

Vector $\boldsymbol{U}^{\prime}\left(=\mathrm{O}^{\prime}{ }_{1 \mathrm{c}} \mathrm{O}^{\prime}{ }_{1}\right)$ formed by projecting $\boldsymbol{U}$ on this paper is given by

$$
\boldsymbol{U}^{\prime}=\boldsymbol{U}-\left(\boldsymbol{U} \cdot \boldsymbol{a}_{N}\right) \boldsymbol{a}_{N}
$$

$\widehat{\mathrm{A}_{1} \mathrm{~A}_{2}}$ is divided into $n_{1}$ segments. Then,

$$
w=\frac{R_{1}\left(\alpha_{1}+\alpha_{2}\right)}{n_{1}}
$$

The position of the $n_{0}$-th segment from $A_{2}$ is designated as $\mathrm{C}_{n 0}$. Then the amount of interference $\delta$ at this segment is expressed as

$$
\delta=\left|\boldsymbol{U}^{\prime}\right| \cos \psi-g_{1}
$$

Contact force $q$ is obtained by substituting $w$ and $\delta$ obtained from Eqs. (41) and (42) into Eq. (37). Unit vector $\boldsymbol{a}_{U}$ in the $\boldsymbol{U}^{\prime}$ direction and vector $\boldsymbol{a}_{H}$ perpendicular to $\boldsymbol{a}_{U}$ and $\boldsymbol{a}_{N}$ are expressed as

$$
\begin{aligned}
& \boldsymbol{a}_{U}=\boldsymbol{U}^{\prime} /\left|\boldsymbol{U}^{\prime}\right| \\
& \boldsymbol{a}_{H}=\boldsymbol{a}_{N} \times \boldsymbol{a}_{U}
\end{aligned}
$$

Suppose $\boldsymbol{a}_{R}$ denotes a unit vector on $\overline{\mathrm{O}_{1}^{\prime} \mathrm{C}_{n 0}}$. Then,

$$
\boldsymbol{a}_{R}=\boldsymbol{a}_{U} \cos \psi+\boldsymbol{a}_{H} \sin \psi
$$

Force $\boldsymbol{F}_{1}$ acting on the cage from the inner race is expressed as

$$
\boldsymbol{F}_{1}=\sum_{j=1}^{n} \sum_{n_{0}=1}^{n_{1}} q \boldsymbol{a}_{R}
$$

Velocity of the inner race $\boldsymbol{V}_{1}$ and cage velocity $\boldsymbol{V}_{c}$ at point $\mathrm{C}_{n o}$ are expressed as

$$
\begin{aligned}
& \boldsymbol{V}_{1}=\omega_{1} \times R_{1} \boldsymbol{a}_{R}+\dot{\boldsymbol{E}}_{1} \\
& \boldsymbol{V}_{c}=\omega_{c} \times\left(\boldsymbol{O}_{1}+\boldsymbol{E}_{1}+R_{1} \boldsymbol{a}_{R}-\boldsymbol{G}_{c}-\boldsymbol{E}_{G c}\right)+\dot{\boldsymbol{E}}_{G c}
\end{aligned}
$$

Unit vector in the direction of $\boldsymbol{V}_{1}-\boldsymbol{V}_{c}$, the inner race relative velocity vector with regard to the cage at the point of $\mathrm{C}_{n o}$, is given by

$$
\boldsymbol{a}_{V}=\frac{\boldsymbol{V}_{1}-\boldsymbol{V}_{c}}{\left|\boldsymbol{V}_{1}-\boldsymbol{V}_{c}\right|}
$$

The frictional force acting on segment $w$ at the point $\mathrm{C}_{n 0}$ is given by $\sigma q \boldsymbol{a}_{V}$. Therefore, frictional force $\boldsymbol{H}_{1}$ acting from the inner race on the cage is given by

$$
\boldsymbol{H}_{1}=\sigma \sum_{j=1}^{n} \sum_{n_{0}=1}^{n_{1}} q \boldsymbol{a}_{V}
$$

The moment of the frictional force is given by

$$
\boldsymbol{m}_{H_{1}}=\sigma R_{1} \sum_{j=1}^{n} \sum_{n_{0}=1}^{n_{1}} q \boldsymbol{a}_{R} \times \boldsymbol{a}_{V}
$$

For conditions other than those used in Fig. 7, $\boldsymbol{F}_{1}, \boldsymbol{F}_{2}$, $\boldsymbol{H}_{1}, \boldsymbol{H}_{2}, \boldsymbol{m}_{H 1}$, and $\boldsymbol{m}_{H 2}$ can be expressed in the form of Eqs. (46), (50) and (51) in a similar manner to that mentioned above.

\section{Equations of Motion}

\subsection{Motions of inner race and inner race axis}

5.1.1 General expression Figure 8 shows the displacement vector of the inner race and the inner race axis built into it. The end of the axis $\mathrm{O}_{\mathrm{a}}$ lies on the straight line that forms $-\theta$ with regard to the $x$-axis on the $x y$ plane. It is assumed that torsional moment $T_{1} \boldsymbol{a}_{T 1}^{\prime}$, bending moment $\boldsymbol{M}_{1 a}$ and resultant force $\boldsymbol{R}$ are applied to point $\mathrm{O}_{\mathrm{a}}$. The point of action of the external force acting on the axis is shifted to $\mathrm{O}_{\mathrm{s}}$ and it is considered that the couple of the 


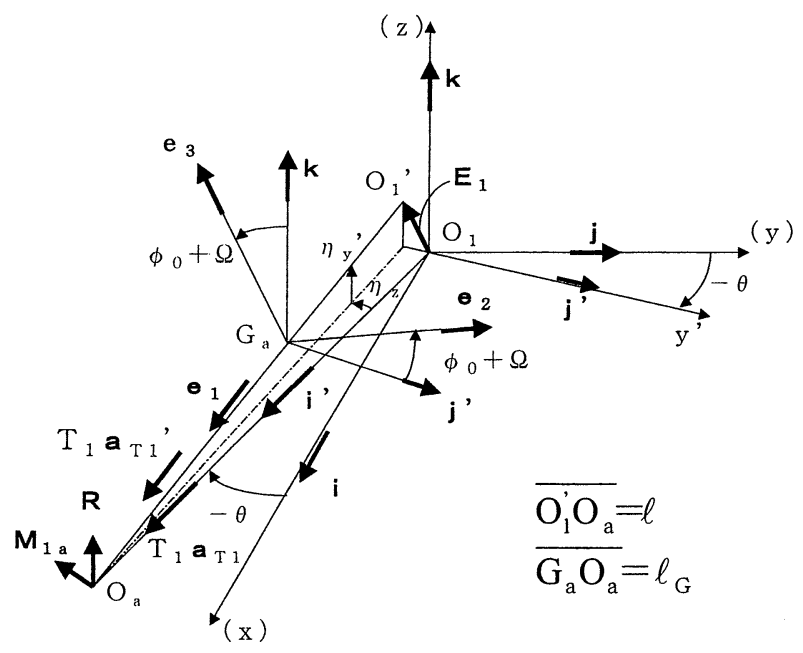

Fig. 8 Displacements of inner race and shaft

force caused by shifting the point of action is included in $\boldsymbol{M}_{1 a}$.

When the inner race and the inner race axis are considered to form one rigid body, the equation of motion about its center of gravity is expressed as

$$
M_{a} \ddot{\boldsymbol{E}}_{G a}=\boldsymbol{R}+\boldsymbol{F}_{0}
$$

in which

$$
\boldsymbol{F}_{0}=\sum_{j=1}^{n}\left(-\boldsymbol{P}_{1 j}-\boldsymbol{P}_{2 j}-\boldsymbol{Q}_{1 j}-\boldsymbol{Q}_{2 j}\right)-\boldsymbol{F}_{1}-\boldsymbol{H}_{1}
$$

As for rotational motion about the center of gravity $G_{a}$, Euler's equations of motion ${ }^{(9),(10)}$ shown below are applied.

$$
\begin{aligned}
& I_{1} \frac{d \omega_{1}}{d t}+\left(I_{3}-I_{2}\right) \omega_{2} \omega_{3}=N_{1} \\
& I_{2} \frac{d \omega_{2}}{d t}+\left(I_{1}-I_{3}\right) \omega_{3} \omega_{1}=N_{2} \\
& I_{3} \frac{d \omega_{3}}{d t}+\left(I_{2}-I_{1}\right) \omega_{1} \omega_{2}=N_{3}
\end{aligned}
$$

in which $I_{1}, I_{2}$ and $I_{3}$ are the moments of inertia relating to the principal axes of inertia selected as the peculiar coordinates of the rigid body, $\omega_{1}, \omega_{2}$ and $\omega_{3}$ are angular velocities around the said principal axes of inertia, and $N_{1}$, $N_{2}$, and $N_{3}$ are the moment components relating to the said peculiar coordinates.

Peculiar coordinates of the rigid body have their origin at the center of gravity and have the fundamental vectors $\boldsymbol{e}_{1}, \boldsymbol{e}_{2}$, and $\boldsymbol{e}_{3}$ shown in Fig. 8.

$$
\begin{aligned}
& \boldsymbol{e}_{1}=\boldsymbol{a}_{T 1}^{\prime}=\boldsymbol{A}_{z}(-\theta) \boldsymbol{A}_{y}\left(\eta_{y^{\prime}}\right) \boldsymbol{A}_{z}\left(\eta_{z}\right) \boldsymbol{i} \\
& \boldsymbol{e}_{2}=\boldsymbol{A}_{z}(-\theta) \boldsymbol{A}_{y}\left(\eta_{y^{\prime}}\right) \boldsymbol{A}_{z}\left(\eta_{z}\right) \boldsymbol{A}_{x}\left(\phi_{0}+\Omega\right) \boldsymbol{j} \\
& \boldsymbol{e}_{3}=\boldsymbol{A}_{z}(-\theta) \boldsymbol{A}_{y}\left(\eta_{y^{\prime}}\right) \boldsymbol{A}_{z}\left(\eta_{z}\right) \boldsymbol{A}_{x}\left(\phi_{0}+\Omega\right) \boldsymbol{k}
\end{aligned}
$$

Angular velocity components $\omega_{1}, \omega_{2}$ and $\omega_{3}$ in the direction of the fundamental vectors are given by

$$
\begin{aligned}
& \omega_{1}=\omega+\dot{\Omega} \\
& \omega_{2}=\dot{\eta}_{y^{\prime}} \cos \left(\phi_{0}+\Omega\right)+\dot{\eta}_{z} \sin \left(\phi_{0}+\Omega\right)
\end{aligned}
$$

$$
\omega_{3}=-\dot{\eta}_{y^{\prime}} \sin \left(\phi_{0}+\Omega\right)+\dot{\eta}_{z} \cos \left(\phi_{0}+\Omega\right)
$$

In the rigid body discussed herein, it is obvious that $I_{2}=I_{3}$. It is therefore assumed that $I_{1}=I_{p}$ and $I_{2}=I_{3}=$ $I_{d}$. These relationships and Eqs. (60) - (62) are substituted into Eqs. (54) - (59) and the expressions are rearranged to obtain the following equations.

$$
\begin{aligned}
& I_{p} \dot{\omega}_{1}=\boldsymbol{N} \cdot a_{T 1}^{\prime} \\
& I_{d} \ddot{\eta}_{y^{\prime}}+I_{p} \omega_{1} \dot{\eta}_{z}=\boldsymbol{N} \cdot\left(\boldsymbol{j}^{\prime}-\eta_{z} \boldsymbol{i}^{\prime}\right) \\
& I_{d} \ddot{\eta}_{z}-I_{p} \omega_{1} \dot{\eta}_{y^{\prime}}=\boldsymbol{N} \cdot\left(\boldsymbol{k}+\eta_{y^{\prime}} \boldsymbol{i}^{\prime}\right)
\end{aligned}
$$

Suppose $\overline{\mathrm{O}_{1} \mathrm{O}_{2}}=l$ and $\overline{\mathrm{G}_{\mathrm{a}} \mathrm{O}_{\mathrm{a}}}=l_{\mathrm{G}}$, the moment relating to the center of gravity $G_{a}$ of the shaft and the inner race is

$$
\begin{aligned}
\boldsymbol{N}= & l_{G} \boldsymbol{a}_{T 1}^{\prime} \times \boldsymbol{R}-\left\{\left(l-l_{G}\right) \boldsymbol{a}_{T 1}^{\prime}+\boldsymbol{O}_{1}+\boldsymbol{E}_{1}\right\} \\
& \times \boldsymbol{F}_{0}+\boldsymbol{N}_{1}+T_{1} \boldsymbol{a}_{T 1}^{\prime}+\boldsymbol{M}_{1 a}
\end{aligned}
$$

in which $N_{1}$ is the moment relating to origin $\mathrm{O}$, generated by the force acting on the inner race in the ball-type constant-velocity joint and is given by

$$
\begin{aligned}
\boldsymbol{N}_{1}= & -\sum_{j=1}^{n} \sum_{k=1}^{2}\left[\left(\boldsymbol{B}_{j}+\boldsymbol{E}_{B j}\right) \times \boldsymbol{P}_{k j}+\left(\boldsymbol{B}_{j}+\boldsymbol{E}_{B j}+\boldsymbol{r}_{k j}\right) \times \boldsymbol{Q}_{k j}\right. \\
& \left.+\boldsymbol{m}_{d k j}\right]-\left(\boldsymbol{O}_{1}+\boldsymbol{E}_{1}\right) \times\left(\boldsymbol{F}_{1}+\boldsymbol{H}_{1}\right)-\boldsymbol{m}_{H 1}
\end{aligned}
$$

$\boldsymbol{R}$ obtained from Eq. (52) is substituted into Eq. (66), and the expression is rearranged as

$$
\left\{\begin{array}{l}
\boldsymbol{N}=l_{G} M_{a} \boldsymbol{a}_{T 1}^{\prime} \times \ddot{\boldsymbol{E}}_{G a}+\boldsymbol{N}_{a} \\
\boldsymbol{N}_{a}=-\left(l \boldsymbol{a}_{T 1}^{\prime}+\boldsymbol{O}_{1}+\boldsymbol{E}_{1}\right) \times \boldsymbol{F}_{0}+\boldsymbol{N}_{1}+T_{1} \boldsymbol{a}_{T 1}^{\prime}+\boldsymbol{M}_{1 a}
\end{array}\right.
$$

Substituting Eqs. (60), (68) into Eq. (63),

$$
I_{p} \ddot{\Omega}=-\left[\left(\boldsymbol{O}_{1}+\boldsymbol{E}_{1}\right) \boldsymbol{F}_{\mathbf{0}} \boldsymbol{a}_{T 1}^{\prime}\right]+\boldsymbol{N}_{1} \cdot \boldsymbol{a}_{T 1}^{\prime}+T_{1}
$$

Substituting Eqs. (60), (68) into Eqs. (64), (65), and omitting minor terms,

$$
\begin{aligned}
& I_{d} \ddot{\eta}_{y^{\prime}}+I_{p} \omega \dot{\eta}_{z}=l_{G} M_{a}\left[\boldsymbol{a}_{T 1}^{\prime} \ddot{\boldsymbol{E}}_{G a} \boldsymbol{j}^{\prime}\right]+\boldsymbol{N}_{a} \cdot\left(\boldsymbol{j}^{\prime}-\eta_{z} \boldsymbol{i}^{\prime}\right) \\
& I_{d} \ddot{\eta}_{z}-I_{p} \omega \dot{\eta}_{y^{\prime}}=l_{G} M_{a}\left[\boldsymbol{a}_{T 1}^{\prime} \ddot{E}_{G a} \boldsymbol{k}\right]+\boldsymbol{N}_{a} \cdot\left(\boldsymbol{k}+\eta_{y^{\prime}} \boldsymbol{i}^{\prime}\right)
\end{aligned}
$$

The following description deals with two cases: in one case, the universal joint of the object under analysis is of plunging type and in the other case, it is of fixed type.

5.1.2 Plunging-type universal joint The end of the inner race axis $\mathrm{O}_{a}$ is considered to be a fixed point pivotally supported by a fixed-type universal joint. Since $\mathrm{O}_{\mathrm{a}}$ is a fixed point, the following expressions are obtained.

$$
\begin{aligned}
& \boldsymbol{E}_{G a}=l_{G} \boldsymbol{i}^{\prime}-l_{G} \boldsymbol{a}_{T 1}^{\prime} \\
& \ddot{\boldsymbol{E}}_{G a}=-l_{G}\left(\ddot{\eta}_{z} \boldsymbol{j}^{\prime}-\ddot{\eta}_{y^{\prime}} \boldsymbol{k}\right)
\end{aligned}
$$

Substituting Eq. (73) into Eqs. (70), (71), and utilizing the following relationship,

$$
I_{a}=I_{d}+M_{a} l_{G}^{2}
$$

Then, 


$$
\begin{aligned}
& I_{a} \ddot{\eta}_{y^{\prime}}+I_{p} \omega \dot{\eta}_{z}=N_{a} \cdot\left(\dot{j}^{\prime}-\eta_{z} i^{\prime}\right) \\
& I_{a} \ddot{\eta}_{z}-I_{p} \omega \dot{\eta}_{y^{\prime}}=\boldsymbol{N}_{a} \cdot\left(\boldsymbol{k}+\eta_{y^{\prime}} \boldsymbol{i}^{\prime}\right)
\end{aligned}
$$

$\boldsymbol{E}_{1}$ has the following conditions:

$$
\boldsymbol{E}_{1} \cdot \boldsymbol{i}^{\prime}=0
$$$$
\boldsymbol{E}_{1} \cdot \boldsymbol{k}=\eta_{y^{\prime}} l
$$

$-\boldsymbol{E}_{1} \cdot \boldsymbol{j}^{\prime}=\eta_{z} l$

Eliminating $\eta_{y^{\prime}}$ and $\eta_{z}$ in Eqs. (75) and (76) by utilizing these expressions, the following differential equations are obtained.

$$
\begin{aligned}
& I_{a} \ddot{E}_{1 z}=I_{p} \omega \sec \theta \dot{E}_{1 y}+l \boldsymbol{N}_{a} \cdot\left(j^{\prime}+\frac{E_{1 y}}{l} \sec \theta \boldsymbol{i}^{\prime}\right) \\
& I_{a} \ddot{E}_{1 y}=-I_{p} \cos \theta \dot{E}_{1 z}-l \cos \theta \boldsymbol{N}_{a} \cdot\left(\boldsymbol{k}+\frac{E_{1 z}}{l} \boldsymbol{i}^{\prime}\right)
\end{aligned}
$$

5.1.3 Fixed-type universal joint It is considered that the end of the inner race axis $\mathrm{O}_{\mathrm{a}}$ is supported by a plunging-type universal joint, and therefore is possible to move in the $\boldsymbol{a}_{p}$ direction. Suppose $\boldsymbol{E}_{p}$ denotes the displacement in the $\boldsymbol{a}_{p}$ direction. Then the following expressions are established.

$$
\begin{aligned}
& \boldsymbol{E}_{p}=E_{p} \boldsymbol{a}_{p} \\
& \boldsymbol{E}_{p} \cdot \boldsymbol{a}_{T 1}^{\prime}=\boldsymbol{E}_{1} \cdot \boldsymbol{a}_{T 1}^{\prime} \\
& \boldsymbol{E}_{G a}=\boldsymbol{E}_{p}+\frac{l_{G}}{l}\left(\boldsymbol{E}_{1}-\boldsymbol{E}_{p}\right) \\
& \eta_{y^{\prime}}=\frac{1}{l}\left(\boldsymbol{E}_{1}-\boldsymbol{E}_{p}\right) \cdot \boldsymbol{k} \\
& \eta_{z}=-\frac{1}{l}\left(\boldsymbol{E}_{1}-\boldsymbol{E}_{p}\right) \cdot \boldsymbol{j}^{\prime}
\end{aligned}
$$

We introduce the following expression.

$$
\frac{\boldsymbol{a}_{p}}{\boldsymbol{a}_{p} \cdot \boldsymbol{i}^{\prime}}=\boldsymbol{b}
$$

Assuming $\boldsymbol{a}_{T 1}^{\prime} \fallingdotseq \boldsymbol{i}^{\prime}$, then from Eqs. (82) and (83),

$$
\boldsymbol{E}_{p} \fallingdotseq\left(\boldsymbol{E}_{1} \cdot \boldsymbol{i}^{\prime}\right) \boldsymbol{b}
$$

Therefore, from Eqs. (84) - (86),

$$
\begin{aligned}
& \boldsymbol{E}_{G a}=\frac{l_{G}}{l} \boldsymbol{E}_{1}+\left(1-\frac{l_{G}}{l}\right)\left(\boldsymbol{E}_{1} \cdot \boldsymbol{i}^{\prime}\right) \boldsymbol{b} \\
& \eta_{y^{\prime}}=\frac{1}{l}\left[\boldsymbol{E}_{1}-\left(\boldsymbol{E}_{1} \cdot \boldsymbol{i}^{\prime}\right) \boldsymbol{b}\right] \cdot \boldsymbol{k} \\
& \eta_{z}=-\frac{1}{l}\left[\boldsymbol{E}_{1}-\left(\boldsymbol{E}_{1} \cdot \boldsymbol{i}^{\prime}\right) \boldsymbol{b}\right] \cdot \boldsymbol{j}^{\prime}
\end{aligned}
$$

Assuming $\boldsymbol{a}_{T 1}^{\prime} \fallingdotseq \boldsymbol{i}^{\prime}$ in Eqs. (70) and (71),

$$
\begin{aligned}
& I_{a} \ddot{\eta}_{y^{\prime}}+I_{p} \omega \dot{\eta}_{z}=-l_{G} M_{a} \ddot{\boldsymbol{E}}_{G a} \cdot \boldsymbol{k}+\boldsymbol{N}_{a} \cdot\left(\boldsymbol{j}^{\prime}-\eta_{z} \boldsymbol{i}^{\prime}\right) \\
& I_{d} \ddot{\eta}_{z}-I_{p} \omega \dot{\eta}_{y^{\prime}}=l_{G} M_{a} \ddot{\boldsymbol{E}}_{G a} \cdot \boldsymbol{j}^{\prime}+\boldsymbol{N}_{a} \cdot\left(\boldsymbol{k}+\eta_{y^{\prime}} \boldsymbol{i}^{\prime}\right)
\end{aligned}
$$

Substituting Eqs. (89), (90), (91) into $\ddot{\boldsymbol{E}}_{G a}, \ddot{\eta}_{y^{\prime}}, \ddot{\eta}_{z}$ in Eqs. (92), (93), and rearranging using Eq. (74),

$$
\begin{aligned}
- & \left(\frac{I_{a}}{l}-l_{G} M_{a}\right)(\boldsymbol{b} \cdot \boldsymbol{k}) \cos \theta \ddot{E}_{1 x} \\
& +\left(\frac{I_{a}}{l}-l_{G} M_{a}\right) \boldsymbol{b} \cdot \boldsymbol{k} \sin \theta \ddot{E}_{1 y}+\frac{I_{a}}{l} \ddot{E}_{1 z} \\
= & -I_{p} \omega \dot{\eta}_{z}+\boldsymbol{N}_{a} \cdot\left(\boldsymbol{j}^{\prime}-\eta_{z} \boldsymbol{i}^{\prime}\right)
\end{aligned}
$$

$$
\begin{aligned}
& {\left[-\frac{I_{a}}{l} \sin \theta+\left(\frac{I_{a}}{l}-l_{G} M_{a}\right)\left(\boldsymbol{b} \cdot \boldsymbol{j}^{\prime}\right) \cos \theta\right] \ddot{E}_{1 x}} \\
& \quad+\left[-\frac{I_{a}}{l} \cos \theta-\left(\frac{I_{a}}{l}-l_{G} M_{a}\right)\left(\boldsymbol{b} \cdot \boldsymbol{j}^{\prime}\right) \sin \theta\right] \ddot{E}_{1 y} \\
& =I_{p} \omega \dot{\eta}_{y^{\prime}}+\boldsymbol{N}_{a} \cdot\left(\boldsymbol{k}+\eta_{y^{\prime}} \boldsymbol{i}^{\prime}\right)
\end{aligned}
$$

If it is assumed that the $\boldsymbol{a}_{p}$ direction component of force $\boldsymbol{R}$ acting on the axis end $\mathrm{O}_{\mathrm{s}}$ is negligibly small, we obtain $\boldsymbol{R} \cdot \boldsymbol{a}_{p} \fallingdotseq 0$. Applying this condition to Eq. (52),

$$
M_{a} \ddot{\boldsymbol{E}}_{G a} \cdot \boldsymbol{a}_{p}=\boldsymbol{F}_{0} \cdot \boldsymbol{a}_{p}
$$

Substituting Eq. (96) into Eq. (89) and expanding it with regard to the element of $\ddot{\boldsymbol{E}}_{1}$,

$$
\begin{aligned}
& \frac{M_{a}}{l}\left[l_{G}\left(\boldsymbol{i} \cdot \boldsymbol{a}_{p}\right)+\left(l-l_{G}\right)\left(\boldsymbol{b} \cdot \boldsymbol{a}_{p}\right) \cos \theta\right] \ddot{E}_{1 x} \\
& \quad+\frac{M_{a}}{l}\left[l_{G}\left(\boldsymbol{j} \cdot \boldsymbol{a}_{p}\right)-\left(l-l_{G}\right)\left(\boldsymbol{b} \cdot \boldsymbol{a}_{p}\right) \sin \theta\right] \ddot{E}_{1 y} \\
& \quad+\frac{l_{G}}{l} M_{a}\left(\boldsymbol{k} \cdot \boldsymbol{a}_{p}\right) \ddot{E}_{1 z}=\boldsymbol{F}_{0} \cdot \boldsymbol{a}_{p}
\end{aligned}
$$

$\eta_{y^{\prime}}$ and $\dot{\eta}_{y^{\prime}}$, and $\eta_{z}$ and $\dot{\eta}_{z}$ on the right-hand side of Eqs. (94) and (95) are obtained from Eqs. (90) and (91).

\subsection{Motion of cage}

The motion of the center of gravity of the cage $G_{c}$ can be expressed by

$$
\boldsymbol{M}_{c} \ddot{\boldsymbol{E}}_{G c}=\sum_{j=i}^{n} \sum_{k=5}^{7}\left(\boldsymbol{P}_{k j}-\boldsymbol{Q}_{k j}\right)+\boldsymbol{F}_{1}+\boldsymbol{H}_{1}-\boldsymbol{F}_{2}-\boldsymbol{H}_{2} \text { (98) }
$$

Equations (54) - (56) are applied to the rotational motion about the center of gravity. Figure 9 shows the components of the rotational displacement $\xi$ and the fundamental vectors relating to the peculiar coordinates of the rigid body. The fundamental vectors are given by

$$
\begin{aligned}
& \boldsymbol{e}_{1}=\boldsymbol{A}_{y}\left(\xi_{y}\right) \boldsymbol{A}_{z}\left(\xi_{z}\right) \boldsymbol{i} \\
& \boldsymbol{e}_{2}=A_{y}\left(\xi_{y}\right) \boldsymbol{A}_{z}\left(\xi_{z}\right) \boldsymbol{A}_{x}\left(\phi_{c}\right) \boldsymbol{j} \\
& \boldsymbol{e}_{3}=\boldsymbol{A}_{y}\left(\xi_{y}\right) \boldsymbol{A}_{z}\left(\xi_{z}\right) \boldsymbol{A}_{x}\left(\phi_{c}\right) \boldsymbol{k}
\end{aligned}
$$

Angular velocity components $\omega_{1}, \omega_{2}$ and $\omega_{3}$ in the directions of $\boldsymbol{e}_{1}, \boldsymbol{e}_{2}$, and $\boldsymbol{e}_{3}$, respectively, are given by

$$
\begin{aligned}
& \omega_{1}=\dot{\phi}_{c} \\
& \omega_{2}=\dot{\xi}_{y} \cos \phi_{c}+\dot{\xi}_{z} \sin \phi_{c}
\end{aligned}
$$

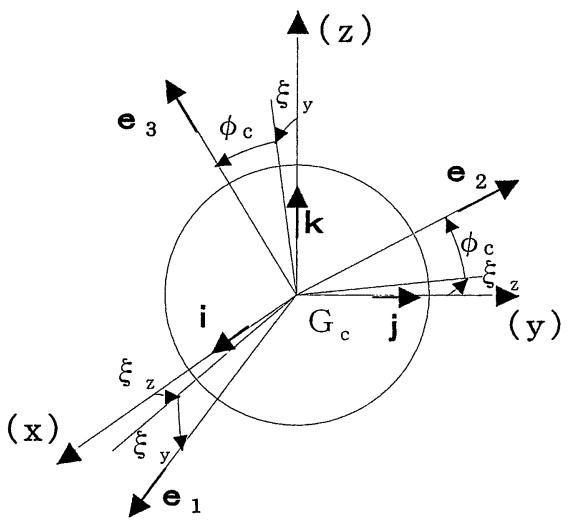

Fig. 9 Angular displacements of cage 
$\omega_{3}=-\dot{\xi}_{y} \sin \phi_{c}+\dot{\xi}_{z} \cos \phi_{c}$

In Eqs. (54)-(56), assuming $I_{1}=I_{c p}, I_{2}=I_{3}=I_{c d}$, substituting Eqs. (102) - (104) and rearranging the expressions, we obtain

$$
\begin{aligned}
& I_{c p} \ddot{\phi}_{c}=\boldsymbol{N}_{c} \cdot\left(\boldsymbol{i}+\xi_{z} \boldsymbol{j}-\boldsymbol{\xi}_{y} \boldsymbol{k}\right) \\
& I_{c d} \ddot{\xi}_{y}+I_{c p} \dot{\phi}_{c} \dot{\xi}_{z}=\boldsymbol{N}_{c} \cdot\left(\boldsymbol{j}-\xi_{z} \boldsymbol{i}\right) \\
& I_{c d} \ddot{\xi}_{z}-I_{c p} \dot{\phi}_{c} \dot{\xi}_{y}=\boldsymbol{N}_{c} \cdot\left(\boldsymbol{k}+\xi_{y} \boldsymbol{i}\right)
\end{aligned}
$$

in which $\boldsymbol{N}_{c}$ denotes the moment relating to the center of gravity of the cage and is written as

$$
\begin{aligned}
\boldsymbol{N}_{c}= & \sum_{j=1}^{n}\left(\boldsymbol{B}_{j}+\boldsymbol{E}_{B j}-\boldsymbol{G}_{c}-\boldsymbol{E}_{G c}\right) \times\left(\boldsymbol{P}_{5 j}+\boldsymbol{P}_{6 j}+\boldsymbol{P}_{7 j}\right) \\
& +\left(\boldsymbol{O}_{1}+\boldsymbol{E}_{1}-\boldsymbol{G}_{c}-\boldsymbol{E}_{G c}\right) \times\left(\boldsymbol{F}_{1}+\boldsymbol{H}_{1}\right)+\boldsymbol{m}_{H 1} \\
& +\left[\boldsymbol{O}_{2}-\boldsymbol{G}_{c}+\boldsymbol{\xi} \times\left(\boldsymbol{O}_{2}-\boldsymbol{G}_{c}\right)\right] \times\left(-\boldsymbol{F}_{2}-\boldsymbol{H}_{2}\right)-\boldsymbol{m}_{H 2} \\
& +\sum_{j=1}^{n}\left\{\sum _ { k = 5 } ^ { 7 } \left[\left(\boldsymbol{B}_{j}+\boldsymbol{E}_{B j}-\boldsymbol{G}_{c}-\boldsymbol{E}_{G c}+\boldsymbol{r}_{k j}\right)\right.\right. \\
& \left.\left.\times\left(-\boldsymbol{Q}_{k j}\right)\right]-\boldsymbol{m}_{d 5 j}-\boldsymbol{m}_{d 6 j}-\boldsymbol{m}_{d 7 j}\right\}
\end{aligned}
$$

\subsection{Motion of ball}

The equation of motion about the center of gravity of the ball is given by

$$
\begin{aligned}
& M_{B}\left(\ddot{\boldsymbol{B}}_{j}+\ddot{\boldsymbol{E}}_{B j}\right) \\
& \quad=\boldsymbol{P}_{1 j}+\boldsymbol{P}_{2 j}-\boldsymbol{P}_{3 j}-\boldsymbol{P}_{4 j}-\boldsymbol{P}_{5 j}-\boldsymbol{P}_{6 j}-\boldsymbol{P}_{7 j}+\sum_{k=1}^{7} \boldsymbol{Q}_{k j}
\end{aligned}
$$

The equation of motion about the rotation about the center of gravity of the balls is given by

\begin{tabular}{|c|c|c|c|}
\hline Parts & Joint type & Equation no. & Main variables \\
\hline \multirow{4}{*}{$\begin{array}{l}\text { Inner } \\
\text { race } \\
+ \\
\text { shaft }\end{array}$} & \multirow{2}{*}{ Plunge } & (69) & $\Omega$ \\
\hline & & $(80)(81)$ & $\mathrm{E}_{1 \mathrm{y}}$ \\
\hline & \multirow{2}{*}{ Fixed } & (69) & $\Omega$ \\
\hline & & (94) (95) (97) & $\begin{array}{lll}E_{1 \mathrm{x}} & \mathrm{E}_{1 \mathrm{y}} & \mathrm{E}_{1 \mathrm{z}} \\
\end{array}$ \\
\hline \multirow{2}{*}{ Cage } & & (98) & $E_{G c x} \quad E_{G c y} \quad E_{G c z}$ \\
\hline & & $(105)(106)(107)$ & $\phi_{\mathrm{c}}{ }^{(*)} \xi_{\mathrm{y}}$ \\
\hline \multirow{2}{*}{ Ball } & & $(109)$ & $E_{B x j} \quad E_{B y j} \quad E_{B z j}$ \\
\hline & & $(110)$ & $\omega_{\mathrm{Bx} j} \quad \omega_{\mathrm{By} j} \quad \omega_{\mathrm{Bz} j}$ \\
\hline
\end{tabular}

$$
I_{B} \dot{\boldsymbol{\omega}}_{B j}=\sum_{k=1}^{7} \boldsymbol{r}_{k j} \times \boldsymbol{Q}_{k j}+\sum_{k=1}^{7} \boldsymbol{m}_{d k j}
$$

\section{Simultaneous Ordinary Differential Equations}

The independent differential equations derived from the equations of motion are summarized in Table 2 . These constitute the simultaneous ordinary differential equations in which time $t$ acts as the independent variable. In the equations, $\boldsymbol{P}_{k j}, \boldsymbol{Q}_{k j}, \boldsymbol{m}_{d k j}, \boldsymbol{F}_{m}, \boldsymbol{H}_{m}$, and $\boldsymbol{m}_{H m}$ are given

Table 2 Differential equations

(*) $\phi_{\mathrm{C}}$ is coincident with $\phi_{\mathrm{c}}($ for $\mathrm{j}=1)$ by equations such as (7), (18), (21), (46), (50), (51) as the function of the dependent variables of the differential equations. The number of the equations and of the dependent variables is $9+6 n$ for plunging-type constant-velocity universal joints and $10+6 n$ for fixedtype constant-velocity universal joints. It is possible to solve these simultaneous ordinary differential equations numerically using an electronic computer. At the same time, $\boldsymbol{P}_{k j}, \boldsymbol{Q}_{k j}, \boldsymbol{F}_{m}, \boldsymbol{H}_{m}$, etc. can be obtained together. Rotational speed variation can be assessed in terms of $\dot{\Omega} / \omega$.

The secondary couple induced to the inner and outer race axes is the components perpendicular to the axes of the moment with regard to the origin generated by the forces acting on the inner and outer race of the constantvelocity universal joint.

With regard to the inner race, from Eq. (67), zcomponent $M_{1 z}$ and the component in the $x y$-plane, $M_{1 x y}$, are expressed as

$$
\begin{aligned}
& M_{1 z}=\left(-\boldsymbol{N}_{1}\right) \cdot \boldsymbol{k} \\
& M_{1 x y}=\left(-\boldsymbol{N}_{1}\right) \cdot \boldsymbol{j}^{\prime}
\end{aligned}
$$

For the outer race, the following expressions are obtained.

$$
\begin{aligned}
\boldsymbol{N}_{2}= & \sum_{j=1}^{n} \sum_{k=3}^{4}\left[-\left(\boldsymbol{B}_{j}+\boldsymbol{E}_{B j}\right) \times \boldsymbol{P}_{k j}+\left(\boldsymbol{B}_{j}+\boldsymbol{E}_{B j}+\boldsymbol{r}_{k j}\right)\right. \\
& \left.\times \boldsymbol{Q}_{k j}+\boldsymbol{m}_{d k j}\right]-\left[\boldsymbol{O}_{2}+\boldsymbol{E}_{G c}+\boldsymbol{\xi} \times\left(\boldsymbol{O}_{2}-\boldsymbol{G}_{c}\right)\right] \\
& \times\left(\boldsymbol{F}_{2}+\boldsymbol{H}_{2}\right)-\boldsymbol{m}_{H 2} \\
M_{2 z}= & \boldsymbol{N}_{2} \cdot \boldsymbol{k} \\
M_{2 x y} & =\boldsymbol{N}_{2} \cdot(-\sin \theta \boldsymbol{i}+\cos \theta \boldsymbol{j})
\end{aligned}
$$

Torque $T_{2}$ acting on the outer race axis is given by $T_{2}=N_{2} \cdot a_{T 2}$

\section{Conclusions}

The forces acting on the inside of ball-type constantvelocity joints in which the ball and the track have an angular contact, and the motions of their parts are analyzed based on dynamics taking frictional forces into consideration. The following results were obtained.

(1) The analysis resulted in simultaneous ordinary differential equations in which time is the independent variable and the displacements of each part are the dependent variables.

(2) It is possible to solve these simultaneous ordinary differential equations numerically using an electronic computer. The forces acting on the inside can be obtained together.

It is possible to obtain the secondary couple induced at the inner and outer race axes, the transmitting torque variation and the rotational speed variation from the numerical solutions.

The results of numerical analysis by the simultaneous ordinary differential equations will be presented in a follow-up report ${ }^{(11)}$. 


\section{References}

(1) Kimata, K., Analysis of Ball-Type Constant-Velocity Joints Based on Statics, Transaction of Japan Society of Mechanical Engineers, Vol.58, No.545, C (1992), pp.263-270.

(2 ) Nagatani, H., Static Analysis of the CV-Joints, the Interconnecting Shaft, and the Support Bearing System, Transaction of Japan Society of Mechanical Engineers, Vol.67, No.655, C (2001), pp.823-832.

( 3 ) Kimata, K., Friction and Lubrication of Constant Velocity Joints, Lubrication, Vol.31, No.10 (1986), pp.697-702.

(4) Constant Velocity Joints for Automobiles, Catalog No.5601-II/JE, (1999), p.2, NTN.

( 5 ) Bearing Lubrication Handbook, (1961), p.230, The Nikkan Kogyo Shimbun.

( 6 ) Harris, T.A., Rolling Bearing Analysis, (1966), p.208,
John Wiley \& Sons, Inc.

( 7 ) Palmgren, A., Grundlagen der Warzlagertechnik, (1962), p.51, FRANCKH'SCHE VERLAGSHANDLUNG STUTTGART.

( 8 ) Harris, T.A., The Effect of Misalignment on the Fatigue Life of Cylindrical Roller Bearings Having Crowned Rolling Members, Trans. ASME, J. of Lub. Tech., Vol.9, No.2 (1969), pp.294-300.

(9) Kunii, S. and Chida, K., Dynamics II, (1958), p.114, Maruzen.

(10) Mechanical Engineering Handbook I, (1951), section 3-p.49, Japan Society of Mechanical Engineers.

(11) Kimata, K., Nagatani, H., Imoto, M. and Kohara, T., Numerical Analysis and Experiments on the Characteristics of Ball-Type Constant-Velocity Joints, Transaction of Japan Society of Mechanical Engineers, Vol.69, No.678, C (2003), pp.487-495. 